EESTI NSV TEADUSTE AKADEEMIA TOIMETISED. XV KÖIDE FOOSIKA-MATEMAATIKA- JA TEHNIKATEADUSTE SEERIA, 1966, NR. 1

ИЗВЕСТИЯ АКАДЕМИИ НАУК ЭСТОНСКОЙ ССР. ТОМ ХV СЕРИЯ ФИЗИКО-МАТЕМАТИЧЕСКИХ И ТЕХНИЧЕСКИХ НАУҚ. 1966, № 1

\title{
АМПЛИТУДНО-ФАЗОВАЯ АХРОМАТИЗАЦИЯ ДИЭЛЕКТРИЧЕСКИХ ИНТЕРФЕРЕНЦИОННЫХ ЗЕРКАЛ
}

Излагается простой метод синтеза многослойных диэлектрических полупрозрачных зеркал (светоделителей), комплексный амплитудный коэффициент отражения которых слабо зависит от длины волны в заданном спектральном интервале.

\section{Введение}

Задача синтеза ахроматических диэлектрических интерференционных светоделителей уже рассматривалась несколькими авторами [ [1-4]. Под ахроматизмом в этих работах понимается требование, чтобы энергетические коэффициенты отражения $R$ и пропускания $D$ светоделителя были приближенно постоянны в заданном спектральном интервале. Однако, как подчеркнул недавно Книттл [5], в некоторых случаях этого недостаточно. Кроме постоянства $R$, нужно, чтобы комплексный амплитудный коэффициент отражения

$$
r=\sqrt{R} e^{i \xi}
$$

тоже слабо зависел от длины волны. Называя вещественную амплитуду $|r|=\sqrt{R}$ просто амплитудой, можем сказать, что речь в этой задаче ндет об ахроматизации отражения по амплитуде и фазе.

В названной работе [5] Қниттл решает эту задачу методом, сходным с развитым им ранее [3] способом одной лишь амплитудной (энергетнческой) ахроматизации. Обе задачи решаются им на основе соответствуюших точных формул теюрии многослойных пленок. Вследствие значительной сложности этих формул, метод Книттла требует довольно громоздких вычислений. Поэтому, несмотря на хорошие результаты, полученные Книттлом, предпочтительным был бы более простой метод.

По мнению автора настоящей статьи, в теории синтеза светоделителей можно обойтись без сложных точных формул. Вместо них можно с успехом применить одпу приближенную формулу. Ее эффективность была уже показана в задаче об энергетической ахроматизации светоделителя в предыдущей статье автора [4]. Тот же метод с небольшим изменением будет применен в настоящей статье к задаче комбинированной амплитудно-фазовой ахроматизации отражения. Қак увидим ниже, фазовая ахроматизапия по этому методу лишь незначительно ухудшает амплитудную ахроматизацию, так что практически качество последней остается неизменным.

В последующем изложении мы будем пользоваться в основном обозначениями статьи [4]. 


\section{Метод}

Итак, будем исходить из приближенной формулы

$$
r=\frac{-\sum_{m=0}^{N} v_{m} e^{-2 i\left(\alpha_{1}+\alpha_{2}+\ldots+\alpha_{m}\right)}}{\sqrt{Q+1}}
$$

где

$$
\begin{gathered}
Q=\left|\sum_{m=0}^{N} v_{m} e^{-2 i\left(\alpha_{1}+\alpha_{2}+\ldots \alpha_{m}\right)}\right|^{2} \\
v_{m}=\frac{1}{2} \ln \frac{n_{m+1}}{n_{m}} \\
\alpha_{m}=k n_{m} h_{m} .
\end{gathered}
$$

Падение света предполагается нормальным; $k=\frac{2 \pi}{\lambda}-$ волновоє число; $h_{m}$ - толщина $m$-го слоя; $n_{0}, n_{1}, n_{2}, \ldots n_{N+1}$ - показатели преломления исходной среды, $N$ слоев и подложки.

Задача заключается в том, чтобы сделать $r$ приближенно постоянным в заданном спектральном интервале. Пусть оптические толщины всех слоев равны

$$
\alpha_{1}=\alpha_{2}=\ldots=\alpha_{N} \equiv \alpha,
$$

причем в середине шкалы частот выбранного спектрального интервала слои пусть будут четвертьволновые, т. е.

$$
\alpha=\frac{\pi}{2} \text {. }
$$

Әти условия довольно обычны; в частности, они применялись ранее почти во всех задачах синтеза ахроматичных пленок [1-6]. Таким образом, проблема сводится к определению необходимых значений показателей преломления слоев.

Формулы (2) и (3) принимают теперь вид:

$$
r=\frac{-\sum_{m=0}^{N} v_{m} e^{-2 i m \alpha}}{\sqrt{Q+1}}
$$

и

$$
Q(\alpha)=\left|\sum_{m=0}^{N} v_{m} e^{-2 i m \alpha}\right|^{2}
$$

Из них следует, что

$$
Q=\frac{R}{1-R}
$$


Отсюда вытекает, что в интересах амплитудной (энергетической) ахрсматизации $Q$ должно мало отклоняться от некоторой заданной величины

$$
Q_{0} \equiv Q\left(\frac{\pi}{2}\right)
$$

Тогда $R$ будет мало отклоняться от значения

$$
R_{\alpha=\frac{\pi}{2}}=\frac{Q_{0}}{Q_{0}+1}
$$

Далее ахроматизация по фазе требует, чтобы величина

$$
q \equiv \sum_{m=0}^{N} v_{m} e^{-2 i m \alpha}
$$

тоже слабо зависела от частоты. Это значит, что величина

$$
\left|\frac{\partial q}{\partial \alpha}\right|^{2}
$$

должна быть возможно мала во всем спектральном интервале. Это условие гарантирует, очевидно, не только фазовую, но и амплитудную ахроматизацию. Таким образом, в качестве критерия фазово-амплитудного ахроматизма можем написать условие

$$
\overline{\left|\frac{\partial q}{\partial \alpha}\right|^{2}}=\frac{1}{\alpha_{2}-\alpha_{1}} \int_{\alpha_{1}}^{\alpha_{2}}\left|\frac{\partial q}{\partial \alpha}\right|^{2} d \alpha=\min
$$

где $\alpha_{1}, \alpha_{2}$ означают границы выбранного спектрального интервала.

Подставляя в формулу (14) выражение (13) для $q$, находим

$$
\frac{1}{\alpha_{2}-\alpha_{1}} \int_{\alpha_{1}}^{\alpha_{2}}\left|\sum_{m=0}^{N} m v_{m} e^{-2 i m \alpha}\right|^{2} d \alpha=\min
$$

или

$$
\sum_{k=1}^{N} \sum_{m=1}^{N} k m v_{k} v_{m} \overline{\cos 2(k-m) \alpha}=\min
$$

Выражение, стоящее в левой части этого равенства, представляет собой квадратичную форму от величин $v_{1}, v_{2}, \ldots v_{N}$ с коэффициентами $\overline{\cos 2(k-m) \alpha}$, зависящими от спектрального интервала. Минимум этой квадратичной формы следует искать с учетом следующих двух дополнительных условий:

$$
v_{0}+v_{1}+\ldots+v_{N}=\frac{1}{2} \ln \frac{n_{N+1}}{n_{0}}=\mathrm{const}
$$

и

$$
v_{0}-v_{1}+-\ldots+(-1)^{N_{v_{N}}}=\operatorname{Arsh} \sqrt{Q_{0}}=\text { const. }
$$


Первое из них вытекает из формулы (4), а второе нужно для того, чтобы выполнялось условие (12), так как точная формула $R$ при $\alpha=\frac{\pi}{2}$ имеет Вид

$$
R_{\alpha=\frac{\pi}{2}}=\operatorname{th}^{2}\left(v_{0}-v_{1}+-\ldots+(-1)^{N} v_{N}\right)
$$

После того, как квадратичная форма (16) с учетом условий (17) и (18) минимизирована и соответствующие минимуму значения $v_{0}$, $v_{1}, \ldots v_{N}$ найдены, то по формуле (4) вычисляются искомые показатели преломления всех слоев.

\section{Пример и обсуждение}

Для иллюстрации описанного метода ограничимся единственным примером. Пусть $N=3, n_{0}=1, n_{N+1}=1,52$ и $Q_{0}=1$ (т. е. $\left.R_{a=\frac{\pi}{2}}=0,50\right)$. Спектральный интервал выберем равным одной октаве:

$$
\frac{\pi}{3} \leqslant a \leqslant \frac{2 \pi}{3}
$$

Тогда получим условие (16) в виде

$$
v_{1}^{2}+4 v_{2}^{2}+9 v_{3}^{2}-\frac{6 \sqrt{3}}{\pi}\left(v_{1} v_{2}+3 v_{2} v_{3}\right)+\frac{9 \sqrt{3}}{2 \pi} v_{1} v_{3}=\min
$$

а дополнительные условия будут:

$$
\left.\begin{array}{l}
v_{0}+v_{1}+v_{2}+v_{3}=0,20936 \\
v_{0}-v_{1}+v_{2}-v_{3}=0,88137
\end{array}\right\}
$$

Процедура минимизирования квадратичной формы приводит к системе линейных уразнений. Не останавливаясь на этом элементарном расчете, прнведем только результат.

Для $v_{0}, v_{1}, v_{2}, v_{3}$ получаются следующие значения:

$$
\left.\begin{array}{l}
v_{0}=0,74979 \\
v_{1}=-0,25681 \\
v_{\varepsilon}=-0,20443 \\
v_{3}=-0,07919 .
\end{array}\right\}
$$

Отсюда находим показатели преломления слоев

$$
\left.\begin{array}{l}
n_{1}=4,480 \\
n_{2}=2,680 \\
n_{3}=1,781
\end{array}\right\}
$$

Остается вычислить $R$ и фазу $\xi$ в зависимости от $\alpha$. Это делается, конечно, по соответствующим точным формулам. Окончательные результаты таковы:

$$
\frac{R}{D}=1,000-0,7846 \cos ^{2} \alpha+5,855 \cos ^{4} \alpha-6,026 \cos ^{6} \alpha
$$




$$
\tan \xi=\frac{\sin 2 \alpha\left(-0,06087+0,3332 \cos ^{2} \alpha-1,0010 \cos ^{4} \alpha\right)}{-1+0,5960 \cos ^{2} \alpha-4,456 \cos ^{4} \alpha+4,708 \cos ^{6} \alpha}
$$

График, вычисленный по этим формулам, нзображен на рисунке.

Итак, мы получили действительно хорошую ахроматичность: разность между крайними значениями $R$ едва превышает $2,5 \%$, а разность между крайними значениями $\xi$ меньше $4^{\circ}$.

Сравним изложенный метод с методом одной лишь энергетической ахроматизации, примененным в статье [4]. Разница между ними состоит только в том, что вместо минимизирования $\sqrt{\left.\frac{\partial q}{\partial \alpha}\right|^{2}}$ мы там минимизировали величину $\overline{\left.\frac{\partial}{\partial \alpha}\left(q e^{-2 i g \alpha}\right)\right|^{2}}$. Добавление множителя $e^{-2 i g \alpha}$ не изменяет абсолютной величины $q$ и поэтому, нарушая фазовую ахроматизацию, не влияет на энергетическую. Цель введения этого множителя в том и состояла, чтобы путем предоставления большей свободы в изменении фазы приобрести дополнительный варьируемый аргумент $g$ в интересах более эффективной ахроматизации амплитуды. Действительно, как показывают соответствующие расчеты, отказ от ахроматизации фазы по методу статьи [ $\left.{ }^{4}\right]$ дает несколько лучшую амплитудную ахроматизацию. Однако разница невелика. Изложенный в настоящей статье метод дает практически столь же хорошую амплитудную ахроматизацию, как и предыдущий, но он дает также и хорошую фазовую ахроматичность. Заметим, однако, еще, что показатель преломления первого слоя, имеющий в обонх случаях особо высокие значения, без фазовой ахроматизации получается все-таки несколько меньшим. Это можно видеть из примеров, приведенных в статье [4].

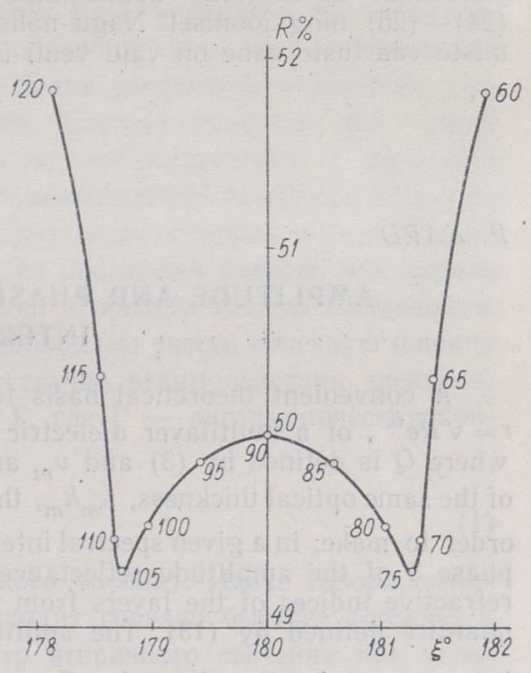

Зависимость $R$ и $\xi$ от $\alpha$. Значения $\alpha$ показаны возле соответствующих точек кривой.

\section{ЛИТЕРАТУРА}

1. Pohlack H., Die Synthese optischer Interferenzschichtsysteme mit vorgegebenen Spektraleigenschaiten, Jenaer Jahrbuch, 1962, 181.

2. Pohla ck H., Zur Theorie der absorptionsfreien achromatischen Lichtteilungsspiegel, Jenaer Jahrbuch, 1956, 79.

3. Knittl Z., K rá 1 M., Optica Acta, 9, 295 (1962).

4. К а рд П., Изв. АН ЭССР. Сер. физ.-матем. и техн. наук, 12, № 1, 3 (1963).

5. Knittl Z., J. phys., 25, No. 1-2, 245 (1964).

6. Ka r d P., Loodus ja matemaatika, 1, 67 (1959). 
P. KARD

\section{DIELEKTRILISTE INTERFERENTSPEEGLITE AKROMATISEERIMINE AMPLITUUDI JA FAASI JARGI}

Mitmekihilise dielektrilise poolläbipaistva kelme amplituudse peegeldumiskoefitsiendi $r=\sqrt{R} e^{i \xi}$ akromatiseerimisel on sobivaks teoreetiliseks aluseks ligikaudne valem (2), kus $Q$ on määratud valemiga (3) ning $v_{m}$ ja $\alpha_{m}$ valemitega (4) ja (5). Eeldades, et kõikide kihtide optilised paksused $n_{m} h_{m}$ on võrdsed, saavad valemid (2) ja (3) lihtsama kuju (8) ja (9). Selleks et kelme amplituudne peegeldumiskoefitsient oleks nii absoluutväärtuselt $\sqrt{R}$ kui ka faasilt $\xi$ antud spektrivahemikus $\alpha_{1} \leqslant \alpha \leqslant \alpha_{2}$ võimalikult konstantne, tuleb: kihtide murdumisnäitajad määrata miinimumtingimusest (14), kus $q$ tähendab valemis (13) defineeritud suurust. Seejuures tuleb arvestada lisatingimusi (17) ja (18), kus $Q_{0}$ en peegeldumiskoefitsiendi $R$ ja läbilaskvuskoefitsiendi $D$ suhte $\frac{R}{D}$ soovitud väärtus ning $n_{0}, n_{N+1}$ on kelmet piiravate keskkondade murdumisnäitajad. Probleem trandub seega: algebraliste lineaarvõrrandite süsteemi lahendamisele. Selle näitena vaadeldakse spektrivahemikus $60^{\circ} \leqslant \alpha \leqslant 120^{\circ}$ kolmekihilist peeglit, millel $Q_{0}=1$. Tulemus esitatakse valemites: (24) - (26) ning joonisel. Nagu näha, on peegli akromaatsus võrdlemisi hea, sest $R$ äärministe väärtuste vahe on vaid veidi üle $2,5 \%$, kuna $\xi$ äärmiste väärtuste vahe ei ületa $4^{\circ}$.

\section{P. KARD}

\section{AMPLITUDE AND PHASE ACHROMATIZATION OF DIELECTRIC INTERFERENCE MIRRORS}

A convenient theoretical basis for the achromatization of the amplitude reflectance, $r=\sqrt{R} e^{i \xi}$, of a multilayer dielectric semireflecting film is the approximate formula (2), where $Q$ is defined by (3) and $v_{m}$ and $\alpha_{m}$ by (4) and (5). Supposing that all layers are of the same optical thickness, $n_{m} h_{m}$, the formulae (2) and (3) get simpler, (8) and (9). In order to make, in a given spectral interval $\alpha_{1} \leqslant \alpha \leqslant \alpha_{2}$, both the absolute value $\overline{V R}$ and thephiase $\xi$ of the amplitude reflectance as constant as possible, one should determine the refractive indices of the layers from the minimum condition (14), where $q$ stands for thequantity defined by (13). The additional conditions (17) and (18) must also be takeir into account. In these formulae $Q_{0}$ means the desired value of the ratio $\frac{R}{D}$ of the reflectance, $R$, and the transmittance, $D$, of the film, and $n_{0}, n_{N+1}$ are the refractive indices of the: bounding media. The whole problem is thus reduced to the solution of a system of linear algebraic equations. An example is given for the three-layer film with $Q_{0}=1$ in the spectral interval $60^{\circ} \leqslant \alpha \leqslant 120^{\circ}$. The results are shown in formulae $(24)-(26)$ and in the figure. As one can see, the achromatism of the mirror is satisfactory, since the difference of the extreme values of $R$ is only slightly more than 2.5 per cent and the difference of the extreme values of $\xi$ does not exceed $4^{\circ}$. 